\title{
LETTER
}

\section{A rat model of glycaemic variability}

\author{
F. Zaccardi • D. Pitocco • G. Ghirlanda
}

Received: 16 March 2009 /Accepted: 16 April 2009/Published online: 14 May 2009

(C) Springer-Verlag 2009

Keywords Animal model of glycaemic variability. Glycaemic variability . Indices of glycaemic variability . Oxidative stress · Vascular dysfunction

\section{Abbreviations \\ PGC Poor glycaemic control \\ UD Untreated diabetic}

To the Editor: There is a growing body of scientific evidence that glycaemic variability is an $\mathrm{HbA}_{1 \mathrm{c}}$-independent predictor of diabetic vascular complications [1]. This conclusion is derived essentially from retrospective epidemiological analyses with hard (microvascular) endpoints [2] and from observational and interventional studies with surrogate endpoints (oxidative stress and endothelial function, assessed with flow-mediated dilation) [3,4]. Clearly, the correlation found between glycaemic variability and microvascular complications/oxidative stress does not necessarily indicate a cause-effect relationship; furthermore, the effect of glycaemic instability on macrovascular disease is not known. As a consequence, whether glycaemic variability is causally linked to diabetic complications and, in particular, to macrovascular dysfunction, remains an open question.

In their elegant paper, Horváth et al. [5] investigated both the effect of glucose fluctuation on endothelial

F. Zaccardi $(\bowtie) \cdot$ D. Pitocco $\cdot$ G. Ghirlanda

Diabetes Care Unit, Policlinico Universitario A. Gemelli,

L.go 'A. Gemelli',

00168 Rome, Italy

e-mail: frazac@fastwebnet.it function (a surrogate marker of macrovascular complications) and the possible mechanisms linking glucose 'swings' to vascular damage. In particular, they compared the effect of glycaemic variability on endothelial dysfunction in four rat models: poor glycaemic control (PGC), good glycaemic control, untreated diabetic (UD) and nondiabetic. Interestingly, although the 'global glycaemic exposition', mathematically expressed by the AUC of their $48 \mathrm{~h}$ glycaemic profiles and correlated with $48 \mathrm{~h}$ mean glucose level [6], was significantly higher in UD rats than in PGC rats, endothelial dysfunction was greater in the PGC rats. This finding supported their conclusion that 'the oscillation of blood glucose level itself is a more important contributor to the loss of endothelial function in diabetes than the average level of mean blood glucose level per se'. In our opinion, this conclusion is not completely supported by the data presented. In fact, the different variability of the glycaemic profiles of PGC versus UD rats is only presumptive and is not supported by an objective mathematical analysis, i.e. comparison of one or more indices of variability (mean amplitude of glycaemic excursions [MAGE], standard deviation, continuous overall net glycaemic action [CONGAn] and others) [6] between the two groups. If the indices of variability for PGC and UD rats were similar, then this would indicate that the better endothelial function of UD rats might be related to an unexplored variable. Therefore, the conclusion should state only that mean blood glucose is not enough to completely explain vascular dysfunction.

Duality of interest The authors declare that there is no duality of interest associated with this manuscript. 


\section{References}

1. Brownlee M, Hirsch IB (2006) Glycemic variability: a hemoglobin $\mathrm{A}_{1 \mathrm{c}}$-independent risk factor for diabetic complications. JAMA 295:1707-1708

2. Kilpatrick ES, Rigby AS, Atkin SL (2008) A1C variability and the risk of microvascular complications in type 1 diabetes: data from the Diabetes Control and Complications Trial. Diabetes Care 31:21982202

3. Monnier L, Mas E, Ginet C et al (2006) Activation of oxidative stress by acute glucose fluctuations compared with sustained chronic hyperglycemia in patients with type 2 diabetes. JAMA 295:1681-1687

4. Ceriello A, Esposito K, Piconi L et al (2008) Oscillating glucose is more deleterious to endothelial function and oxidative stress than mean glucose in normal and type 2 diabetic patients. Diabetes 57:1349-1354

5. Horváth EM, Benkő R, Kiss L et al (2009) Rapid 'glycaemic swings' induce nitrosative stress, activate poly(ADP-ribose) polymerase and impair endothelial function in a rat model of diabetes mellitus. Diabetologia 52:952-961

6. Zaccardi F, Pitocco D, Ghirlanda G (2009) Glycemic risk factors of diabetic vascular complications: the role of glycemic variability. Diabetes Metab Res Rev 25:199-207 